\title{
Kin Selection and Conflict in Seed Maturation
}

\author{
David C. Queller $\dagger$ \\ Division of Biological Sciences, University of Michigan, Ann Arbor, \\ Michigan 48109, U.S.A. \\ (Received 24 February 1982, and in revised form 19 August 1982)
}

There are four genetically distinct components in the developing seeds of flowering plants: maternal sporophyte, gametophyte, êndosperm, and embryo. Each component can potentially influence the quantity or quality of nutrients provided to the embryo of its seed, thereby reducing the amount available to embryos in other seeds of that plant. The theory of kin selection predicts that each component will be selected to favor its own embryo over the other embryos to the extent that it is more closely related to its own. Under this criterion, an embryo should be selected to try to acquire more nutrients than the endosperm should be selected to provide, the endosperm should try to supply more than the gametophyte should, and the gametophyte more than the parent sporophyte. Evidence for this conflict of interests is found in the higher frequency of endopolyploidy, nutrient-absorbing haustoria, and food storage tissues in the embryo and endosperm than in the gametophyte of maternal tissues.

This theory also suggests how the gametophyte, which is the nurse tissue of gymnosperm seeds, was displaced from this role in the flowering plants by an endosperm initiated by a secondary fertilization. "Neoteny" in the pro-angiosperms created conditions in which (1) an endosperm initiated by double fertilization would be more closely related to the embryo than is the gametophyte and (2) the endosperm would be formed early enough to be of significant aid to the embryo.

If this theory is correct it (1) requires a different approach to the study of seed morphology and physiology, (2) increases the plausibility of arguments that flowering plants are a polyphyletic group, (3) provides evidence that parents cannot always control the outcome of conflict with their offspring, and (4) forges a conceptual link in our understanding of the evolution of social interactions in plants and animals.

\section{Introduction}

In natural selection, an allele can reproduce copies of itself not only by promoting the survival and reproduction of its bearer, but also by promoting the survival and reproduction of its bearer's relatives, because their

\footnotetext{
+ Now at School of Biological Sciences, University of Sussex, Brighton BN1 9QG, England. 
genotypes are likely to include identical copies of the allele (Hamilton, 1964). Selection of alleles for their effects on relatives, termed "kin selection", has been successful in accounting for a wide range of behavior in animals (Wilson, 1975). But it has not been convincingly applied to plants, perhaps because they cannot often reliably identify and aid relatives. I will present evidence below that kin selection best accounts for a number of features of seed development, including the role of the endosperm in flowering plants. If correct, this argument has important consequences for the study of seed anatomy and physiology, for theories of the early evolution of the flowering plants, and for kin selection theory itself.

Seed development differs somewhat in the two extant groups of seed plants, the gymnosperms and the flowering plants or angiosperms (Singh \& Johri, 1972; Bhatnagar \& Johri, 1972). Prior to fertilization the ovules of both include diploid maternal tissues and a haploid gametophyte, the latter from the proliferation of a meiotic daughter cell. The gymnosperm gametophyte is relatively large, usually includes several fertile egg cells, and functions as a nutrient acquiring and storing tissue. Fertilization of eggs by pollen nuclei may produce several embryos, but usually only one matures.

Angiosperm seed development is more complex. The gametophyte is tiny, usually eight-nucleate including a single egg nucleus at one end. Two other nuclei, called polar nuclei, are situated near the center of the gametophyte. Two genetically identical sperm nuclei (from the same pollen grain) participate in fertilization. One fertilizes the egg nucleus to initiate the embryo. The other fertilizes the two polar nuclei and the resulting fusion nucleus proliferates into a triploid tissue called the endosperm. Generally, the remainder of the gametophyte degenerates soon after fertilization and the endosperm performs much of the nutrient acquisition and storage role. Thus, in both gymnosperms and angiosperms, developing seeds include three generations: diploid parent, haploid gametophyte, and diploid embryo. But in angiosperms the gametophyte is reduced and double fertilization produces a fourth distinct genetic entity, the endosperm. The important general differences between angiosperm and gymnosperm seed development are summarized in Table 1.

Charnov (1979) has suggested that double fertilization evolved because it increased the relatedness of the nutritive tissue to its embryo compared to its relatedness to other embryos of the same maternal plant. This paper will develop this hypothesis by (1) expanding the analysis to include all four "relatives" within a seed, (2) deriving and testing some predictions of the expanded hypothesis, (3) exploring possible pathways for the evolution of the endosperm, and (4) discussing some alternative hypotheses. 
TABLE 1

Summary of differences in seed development from ovules of gymnosperms and angiosperms. Some exceptions are discussed in the text

\begin{tabular}{lcc}
\hline & Gymnosperms & Angiosperms \\
\hline $\begin{array}{l}\text { Pre-fertilization growth } \\
\text { Gametophyte }\end{array}$ & Much & Little \\
Fertilization & Many cells, several eggs & 8 cells w $/ 1$ egg \& 2 polar nuclei \\
& Egg(s) + sperm(s) & Egg + sperm $=2 n$ embryo \\
& $=2 n$ embryo(s) & 2 polar nuclei + sperm \\
Post-fertilization growth & Little & $=3 n$ endosperm \\
Nurse tissue & Gametophyte & Much \\
Storage tissue & Gametophyte & Endosperm \\
\hline
\end{tabular}

\section{Kin Selection and Conflict in Seeds}

According to kin selection theory, individuals will be selected to behave as if they valued each relative's fitness according to the probability of sharing genes identical by descent with that relative (Hamilton, 1964). If this is true, selection can lead to conflicts between parents and their offspring (Hamilton, 1964; Trivers, 1974). A parent should not favor any of its offspring on the basis of relatedness since parental alleles all have a $50 \%$ chance of having been transmitted to each offspring. (Terms such as "favor" and "value" are used metaphorically and are not intended to imply choice.) In contrast, an offspring allele is certain of being aided by parental investment in that offspring, while investment in its half-siblings will aid identical copies of the allele only $25 \%$ of the time $(50 \%$ for full sibs). Therefore, offspring alleles that affect the ability to procure food from the parent will be selected to try to acquire more than the parent is selected to give.

The theory of parent-offspring conflict can be expanded to include all four "relatives" in a seed, as shown in Table 2. For each such relative, column 1 gives its coefficient of kinship to the embryo in its own seed. The coefficient of kinship is the probability that alleles randomly drawn from the same locus of two individuals will be identical by descent through a common ancestor (Malecot, 1948). Each "relative" in one seed is also related to embryos in other seeds on the same plant. Column 2 gives the coefficients of kinship for these relationships, assuming that the other cmbryos were fathered by a different pollen parent than the first. If the theory of kin selection is correct, the ratios of these two coefficients (column 4) provide a measure of the degree to which each seed component favors its own embryo over other embryos on the same plant. As before, each embryo should value itself more than the parent values it. The gametophyte 
TABLE 2

Coefficients of kinship for each seed component (rows) to each of three kinds of embryos (columns 1-3): embryo 1 in their own seed, embryo 2 in another seed on the same plant fertilized by a different pollen parent, and embryo 3 in another seed of the same plant fertilized by the same pollen parent as embryo 1. Columns 4 and 5 give kinship ratios, which indicate the extent to which each seed component should favor its own embryo over the other two types of embryos

\begin{tabular}{lccccc}
\hline & Embryo 1 & Fmbryo 2 & Embryo 3 & Ratio 1:2 & Ratio 1:3 \\
\hline Maternal tissue & $\frac{1}{4}$ & $\frac{1}{4}$ & $\frac{1}{4}$ & 1 & 1 \\
Gametophyte & $\frac{1}{2}$ & $\frac{1}{4}$ & $\frac{1}{4}$ & 2 & 2 \\
Endosperm & $\frac{1}{2}$ & $\frac{1}{6}$ & 1 & 3 & 2 \\
Embryo 1 & $\frac{1}{2}$ & $\frac{1}{8}$ & $\frac{1}{4}$ & 4 & 2 \\
\hline
\end{tabular}

and the endosperm are both intermediate, but the endosperm's interests are closer to the embryo's. This does not necessarily mean that the endosperm is on the embryo's side and the gametophyte on the parent's. Each relative has a different range of conditions in which it should favor investment in its own embryo at the expense of other embryos. Since this theory treats the four seed components as four relatives whose interests do not entirely coincide, I will refer to it as the kin conflict theory of seed development.

Several points may require clarification. First, kinship ratios greater than one do not indicate an absolute preference for one's own embryo over others; costs and benefits must also be considered. Hamilton's (1964) rule, that self-sacrificial behavior is selected when $b / c>1 / r$ (where $b$ is the benefit to the beneficiary, $c$ is the cost to the altruist, and $r$ is a measure of their relatedness), is a special case of fitness transfer from the viewpoint of the altruist. Note that the one in the numerator may be viewed as the altruist's relatedness to itself. This formula may be generalized to include the viewpoints of other individuals by explicitly using the relatedness to both donor and recipient: $b / c>r_{c} / r_{b}$, where $r_{c}$ and $r_{b}$ measure the individual's relatedness to the individuals incurring the cost and the benefit respectively (see West Eberhard, 1975). When it is expressed as $b r_{b}>c r_{c}$, it is clear that the criterion for the some party to favor the fitness transfer is that the benefit to its genes in one individual must exceed the cost to its genes in the other. The kinship ratios in Table 2 indicate for each seed component the point at which the cost of not obtaining additional resources 
for its own embryo is compensated by a sufficient benefit to other embryos. This point arrives soonest for the parent (who has no particular "own" embryo) and last for the embryo itself. Seed components with higher ratios should favor more prolonged investment in their own embryo or more investment at a given point in development, but do not favor infinite investment.

Several measures of relatedness have been used in kin selection theories (Michod \& Hamilton, 1980). The most common measure, Wright's coefficient of relationship, is inappropriate for interactants with asymmetrical degrees of inbreeding (Hamilton, 1972), a condition which has some similarity to the double dose of maternal genes in the endosperm. Hamilton's (1972) regression coefficient of relatedness was designed to avoid this defect, but while appropriate for the problem at hand, it is unnecessarily complex. The coefficient of kinship is easier to calculate and the ratios obtained are the same as the corresponding ratios of coefficients of relatedness.

The attribution of independent selective interests to the endosperm, a tissue that never produces offspring, may also require some clarification. It must be remembered that genetic reproduction is more than the production of offspring. If endosperm genes can affect the survival or eventual reproduction of embryos sharing those genes, then they can be selected. The endosperm alleles best represented in future generations will be those that act as if they correctly balance the probabilities of their copies being present in the embryos affected. In never producing offspring, the endosperm is no different from sterile social insect workers, which have been shown to have selective interests that are sometimes different from the queen's (Trivers \& Hare, 1976; Oster, Eschel \& Cohen, 1977).

Several assumptions should also be mentioned. First, I assume these arguments based on relatedness are accurate enough to provide valid predictions. More detailed models of gene frequency change must eventually be constructed. Such models exist for parent-offspring conflict, and while they differ at some points, they have generally validated predictions based on relatedness of the sort that I use below (Parker \& Macnair, 1978, 1979; Macnair \& Parker, 1978, 1979; Stamps, Metcalf \& Krishnan, 1978).

A second important assumption is that seed success is commonly limited by resource availability. If this were not true, there would be no trade-off in the fitness of different embryos and therefore no basis for conflict of interests. However, resource limitation is very common and may be the rule in the flowering plants (Stephenson, 1981; Willson \& Burley, in press).

The argument as developed to this point also assumes that the embryos of each maternal plant are commonly fathered by more than one pollen 
parent. Column 3 of Table 2 shows the coefficient of kinship of each seed component to an embryo with the same pollen parent as its own embryo. The kinship ratios of own embryo to full-sib embryo (column 5) show that the interests of the gametophyte, endosperm, and embryo converge when fertilization is by one pollen parent only and the predictions covering such a case would therefore be different. But even in species with pollen grouped into pollinia, plants are likely to be fertilized by pollinia from different plants, so that the average ratios would still be ranked in the same order as those in column 4 .

A final assumption concerns the capabilities of gametophyte, endosperm, and embryo. It may be that parents are usually able to enforce their interests, either because they are larger and more powerful or simply because, as the source of parental investment, they have control over whether or not to dispense it (Alexander, 1974). But if offspring are able to assess their own condition better than the parent can, it may pay parents to be responsive to offspring signals. When this is true, deception of the parent becomes possible (Trivers, 1974). Because of the possibility of total parental domination, predictions of the kin conflict theory must be regarded as testing a complex of two hypotheses: (1) kin selection theory correctly specifies the reproductive interests of the different seed components, and (2) gametophytes, endosperms, and embryos can sometimes influence the disposition of parental resources to seeds.

\section{Predictions of the Kin Conflict Hypothesis}

It has been difficult to test parent-offspring conflict theory directly because not enough is known to predict the optimal strategies of the participants. Even where these seem quantifiable, as in conflict over sex ratios in the social Hymenoptera (Trivers \& Hare, 1976), other selective pressures may obscure the picture (Alexander \& Sherman, 1977). The problem is severe for confiict over seed nutrients because many factors are known to affect selection for seed size (Baker, 1972) and because there are four points of view to consider instead of two. However, the presence of two additional participants enhances the possibilities for testing predictions based on the relative interests of the various parties.

The principal prediction of the kin conflict theory is that the series of maternal tissue, gametophyte, endosperm, and embryo should reflect increasing interest in the nutrition of the embryo within their seed. There may not be four overt strategies evident in any one species. For example, if maternal plants are successful in achieving their optimal distribution of 
nutrients, then the other three seed components should all be selected to try to obtain more for their embryo. Nevertheless, if many species are examined, and if the success of the different seed components varies between species, the prediction should hold.

A subset of this prediction is the easiest to test. Where the two intermediary tissues, gametophyte and endosperm, both occur, the latter should play the dominant role in nourishing the embryo. This is certainly true; the gametophyte of flowering plants is reduced to a few nuclei and rarely persists long after fertilization (Johri, 1963).

But, if the endosperm has been successful in the flowering plants, why has the gametophyte persisted as the nurse tissue of the gymnosperms? Opportunities for double fertilization must have been numerous since multiple sperm delivery is the rule in gymnosperms (Meeuse, 1963). What must be explained is why the products of secondary fertilization evolved into a special nutritive tissue only in the ancestors of the angiosperms. The kin conflict theory suggests two reasons.

In the flowering plants, fertilization of the egg and polar nuclei by identical sperm nuclei from the same pollen grain (Linskens, 1969) assures high relatedness of the endosperm to its embryo. When the contents of an ovule are fertilized by sperm nuclei from different pollen grains, as may occur in gymnosperms, then any extra nutrients brought into the ovule by an incipient endosperm would often go to an embryo other than its "own". There would be no selective pressure for such an endosperm to fight any harder than the gametophyte for nutrients.

A second reason for the restriction of endosperm to the flowering plants concerns the capability of the endosperm to aid its embryo. At the time of fertilization, the gymnosperm gametophyte is quite large and considerable nutrient acquisition has already taken place (Brink \& Cooper, 1947). Some gymnosperm seeds mature even if separated from the maternal plant after pollination but before fertilization (Favre-Duchartre, 1958). Thus, even if an endosperm would value its embryo more than the gametophyte does, it may appear on the scene too late to be effective in implementing its interests.

The taxonomic distribution of intermediary tissues has been shown to be consistent with the kin conflict theory, but other tests are necessary to probe the interests of the maternal sporophyte and embryo. For this purpose I have examined the distribution of three morphological features: endopolyploidy, invasive food-absorbing structures, and the location of food reserves in mature seeds. The relative interests of the four seed components should also be reflected in physiological phenomena such as the roles of hormones in seed maturation, but these have not been as widely studied. 
Endopolyploidy provides multiple templates for protein synthesis and may therefore provide some indication of the level of metabolic activity in different seed tissues. The kin conflict theory suggests that metabolic activity should be highest in the tissues with the greatest stake in the welfare of the embryo. Nagl's (1978) compilation of endopolyploid angiosperm tissues lists five species with endopolyploidy in maternal seed tissues, eleven for the gametophyte, 25 for the endosperm, and 17 for the embryo. In addition, the highest ploidy levels are found in the endosperm and embryo. The evidence thus confirms the prediction but it is not certain that endopolyploidy indicates any actual conflict of interest. A better case can be made for the other two tests.

Endosperms in species of at least 76 families (compiled from Davis, 1966) develop haustorial outgrowths that invade neighboring tissues and absorb nutrients (Bhatnagar \& Kallarackal, 1980). They sometimes even extrude from the ovule and have frequently been characterized as "aggressive" (Chopra \& Sachar, 1963; Masand \& Kapil, 1966). At a later stage the embryo often develops a suspensor which may also be invasive and specialized for absorbtion (Masand \& Kapil, 1966; frequency data comparable to those for endosperm haustoria are not available). In contrast, extensions of the gametophyte are relatively rare. When they do occur, they generally seem to develop before fertilization when the gametophyte is the only tissue whose interests are opposed to the parent's (Masand \& Kapil, 1966; but see Fagerlind, 1936). I have found no records of invasive haustoria derived from maternal seed tissues although in a few species beaks of the maternal nucellus project into the micropyle (Masand \& Kapil, 1966).

It might be argued that haustoria are simply efficient structures for nutrient translocation rather than tools of conflict, but this would leave several questions unanswered. Why are they concentrated in those seed components with the greatest genetic stake in the embryo, especially in light of the fact that these tissues are the last ones formed? Would not growths into the ovule serve just as well to deliver nutrients? Why are haustoria not required to nourish other developing plant parts, such as flower buds, where there are no genetic differences involved? It is worth noting that invasive growths, also known as haustoria, are used by parasitic plants to take nutrients from their hosts in what is clearly a conflict situation (Kuijt, 1969).

The kin conflict theory also predicts that nutrients should be stored mainly by the seed tissues most related to the embryo. Suppose, for example, that nutrients were stored in the gametophyte. If the endosperm were selected to acquire more nutrients than favored by the gametophyte, 
selection on the latter would lead to less storage in its tissue. If the endosperm evolves to store more as a response, the gametophyte would again decrease its storage. Eventually, the entire storage function would be transferred to the endosperm.

Again, this prediction is generally accurate. Nearly all angiosperms store seed reserves in the endosperm or in embryonic tissues such as the cotyledons. No gametophytic storage occurs and only a few families store some of the seed reserves in a maternal tissue, the perisperm (Masand \& Kapil, 1966). Of course, the absence of the gametophyte in late stages of seed development precludes its serving a storage role. But historically this was the role of the gametophyte, and the scenario above provides an explanation for its virtual disappearance. The species with perisperm storage, though unusual, pose a problem for the kin conflict theory. These could be species in which the parent succeeds in controlling the disposition of nutrients so that storage of "excess" nutrients is not a problem, but I know of no way to test this.

It is also significant that storage solely in the embryo occurs in some angiosperms but not in the gymnosperms (Berlyn, 1972). The same condition that allows the endosperm to make a significant contribution to the accrual of resources, early fertilization, should allow the embryo to do likewise. But if this is so, why has the embryo not replaced the endosperm just as endosperm replaced the gametophyte? The probable answer is that the embryo must develop into a mature plant while the endosperm is free to specialize on nutrient acquisition. The earlier division and development of the endosperm, which is fertilized at about the same time as the embryo (Brink \& Cooper, 1947), is probably a reflection of this difference in developmental constraints.

Three families of flowering plants, the Orchidaceae, Trapaceae, and Podostemaceae, have lost the endosperm (Chopra \& Sachar, 1963) and this could be due to supersedure by the embryo. This seems most likely for the orchids since their embryos do not differentiate before seed germination (Swamy, 1949b) and may therefore be partially freed from developmental constraints preventing early specialization on nutrient acquisition. Whatever the cause of endosperm loss, the kin conflict theory predicts that its functions will be assumed primarily by the embryo. The embryos of all three families are in fact characterized by particularly elaborate, absorptive suspensors (Masand \& Kapil, 1966). There are, however, two possible indications of maternal compensation for endosperm loss, though neither is unequivocal. In the Podostemaceae, a cavity forms in the maternal nucellus to accommodate the growing embryo (Mukkada, 1969) but its nutritive role is not established. In Trapa bispinosa, the nucellus is unusually 
persistent (Ghosh, 1954), but this may indicate nothing more than the absence of an endosperm to rapidly digest it.

If endosperms and embryos sometimes succeed in acquiring resources beyond the maternal optimum, then maternal counterploys should evolve. The erection of barriers to endosperm and embryo growth would be the most obvious morphological manifestation of this. This role has sometimes been attributed to the hypostase, a zone of thick-walled cells that develops in some species at the chalazal end of the ovule (Dnyansagar, 1954). In other species, this is one of the two regions through which extra-ovular haustoria often grow (Masand \& Kapil, 1966). Tracer experiments show that water and nutrients must pass around the hypostase in order to enter the ovule (Coe, 1954). The integuments may also serve in part to limit the growth of the endosperm and embryo.

Maternal strategies are not necessarily limited to restricting nutrient accrual. It has been suggested that seed dormancy may sometimes be maternally imposed against the selective interests of the embryo (Westoby, 1981; Cook, 1981; I. M. Sussex, pers. comm.).

The kin conflict theory also predicts that the endosperm should fight only on the behalf of its own embryo. The relevance of this idea to the absence of the endosperm in gymnosperms has already been noted. However, an exception to the usual angiosperm conformity to this rule is found in the composite endosperm of the Loranthaceae (Johri \& Bhatnagar, 1969). This family has lost the integuments of the ovule so that the pre-fertilization gametophytes may abut. Generally, these gametophytes elongate, often far up into the style, where several may be fertilized. The triploid endosperm nuclei migrate back to the base of the "ovule" and then begin dividing. Usually only one embryo survives but all the endosperms fuse. It is not clear to what extent the extra endosperms contribute to the surviving embryo, but it seems likely that they do. However, in the early post-fertilization stages, each embryo is surrounded by its own endosperm (see figures in Narayana, 1954, 1958, and Prakash, 1960). In addition, an endosperm must be among the few reaching the base of the "ovule" (= nutrient source?) for its embryo to survive (Johri \& Prakash, 1965). Thus, each endosperm seems to compete for its own embryo, but after having lost, aids the winner as the best remaining option.

This next-best-option argument would not suffice for the evolution of an endosperm in multiply-fertilized gymnosperms. In the Loranthaceae it is a question of maintaining structures already evolved; but in the gymnosperms, the positive selection required for replacement of the gametophyte would be absent unless an endosperm could reliably aid its own embryo. 
The kin conflict theory also suggests a number of factors that should influence the likelihood of a species evolving particularly aggressive endosperms or embryos. For example, species in which reproductive success is consistently limited by factors other than nutrient availability should give few indications of aggressive endosperms and embryos. This prediction is difficult to test since it requires detailed ecological and embryological information for a number of species.

Deviations from the usual pattern of relatedness within or between seeds should also affect the amount of conflict expected. When pollen is transferred in groups, neighboring embryos will often have the same father. To the extent that seed resource competition is local in space and time, indications of aggressiveness should be reduced because of increased relatedness between seeds (Table 2; Hamilton, 1964; Kress, 1981). Competition should be less severe in insect-pollinated than wind-pollinated plants, and even less severe in plants with pollen grouped together into pollinia or polyads. Note that while orchids usually have pollinia (Swamy, 1949a), the resulting increase in relatedness does not seem to account for their loss of the endosperm. The increase in relatedness is greater for embryos than endosperms (Table 2), but as noted above, embryos seem to have taken over the role of aggressive nutrient acquisition.

Species with frequent self-fertilization (see Table 3) or apomixis should also experience selection for diminished conflict. In apomictic species, the parent and embryo are genetically identical so there should be no conflict of interests. A complication is introduced by the fact that many of these

\section{TABLE 3}

Coefficients of kinship for each seed component to its own embryo (1), and to another embryo (2) on the same plant, when both embryos result from self-fertilization. The kinship ratios in column 3 indicate less conflict of interest among the seed components than among those of outcrossed species (Table 2). The coefficients reflect only one generation of self-fertiliz ation and all would increase with persistent selfing

\begin{tabular}{lccc}
\hline & Embryo 1 & Embryo 2 & Ratio 1:2 \\
\hline Maternal tissue & $\frac{1}{2}$ & $\frac{1}{2}$ & 1 \\
Gametophyte & $\frac{3}{4}$ & $\frac{1}{2}$ & $\frac{3}{2}$ \\
Endosperm & $\frac{3}{4}$ & $\frac{1}{2}$ & $\frac{3}{2}$ \\
Embryo 1 & $\frac{3}{4}$ & $\frac{1}{2}$ & $\frac{3}{2}$ \\
\hline
\end{tabular}


species still require fertilization to initiate endosperm development (Nygren, 1967). One apomict not requiring fertilization has been compared embryologically with an outcrossing congener (Cooper \& Brink, 1949). For any given embryo size, the apomict averaged fewer endosperm cells. Coupled with the fact that nutrients are pre-stored in the ovules of the apomict, this suggests that an embryo can be adequately nourished with little help from the endosperm when there is no conflict of interest.

Other deviations in the usual pattern of relatedness are created by variations in endosperm composition. Most species have the triploid endosperm described above, but not all do (Johri, 1963). In the Onagraceae and a few species in other families, the endosperm is diploid and genetically identical to the embryo. In some other species the gametophyte is tetrasporic, meaning it derives from all four meiotic daughter cells. There are several tetrasporic variants but there are only two effects on relatedness. First, the endosperm may include nuclei from the secondary oocyte that did not produce the egg. An allele in such a nucleus has no copies identical by descent in "its" egg, but has a $50 \%$ expectation of having a copy in other eggs. Second, tetrasporic species often incorporate more than two maternal nuclei into the endosperm, resulting in ploidy levels of up to 15 . Both of these effects cause tetrasporic endosperms to have less of a genetic stake in their embryos, relative to other embryos, than normal endosperms (see Table 4).

The differences in relatedness suggest that the Onagraceae endosperm type should be most aggressive, tetrasporic endosperms least aggressive,

\section{TABLE 4}

Coefficients of kinship for four different endosperm types to their own embryos (1) and to other embryos fertilized by a different pollen parent (2). Rows 3 and 4 are not intended to represent actual endosperm types but to indicate the two effects of tetraspory on kinship. Row 3 shows the effect of incorporating an additional maternal nucleus that is identical to the egg nucleus. Row 4 shows the effect of replacing a maternal nucleus identical to the egg with a nucleus derived from the opposite meiotic reduction division

\begin{tabular}{lccc}
\hline & Embryo 1 & Embryo 2 & Ratio 1:2 \\
\hline Normal type $(3 n)$ & $\frac{1}{2}$ & $\frac{1}{6}$ & 3 \\
Onagraceae type $(2 n)$ & $\frac{1}{2}$ & $\frac{1}{8}$ & 4 \\
Tetraspory effect $1(4 n)$ & $\frac{1}{1}(3 n)$ & $\frac{3}{16}$ & $\frac{8}{3}$ \\
Tetraspory effect $2(3 n)$ & $\frac{1}{12}$ & $\frac{1}{6}$ & $\frac{5}{2}$ \\
\hline
\end{tabular}


and the normal type intermediate. However, it is possible that tetraspory evolved as a maternal strategy to reduce competitiveness of the endosperm (Willson \& Burley, in press) in which case it would be most likely to evolve in species that had particularly effective endosperms. This possibility would have to be ruled out before an unbiased test could be done. There are also a number of species with more than one endosperm type on the same plant, but they are often situated in different areas of the parent tissue (Hjelmqvist, 1964), again making comparison difficult because of possible positional effects in resource competition.

\section{The Origin of the Endosperm}

The tests described above provide evidence for the importance of conflicts of interest in seed maturation. Using similar reasoning, Charnov (1979) suggested that double fertilization evolved because it enhanced the genetic stake of the nutritive tissue in its own embryo. I will examine this hypothesis in more detail below, particularly the problem of circumventing maternal opposition to the evolution of an aggressive endosperm.

In the absence of a good fossil record it is difficult to test this hypothesis directly, though in principle it is possible to do so. It is possible to determine whether the hypothesis is consistent with what is known of the early evolution of the angiosperms.

There have been many attempts to find homologies in the angiosperm and gymnosperm gametophytes, but without much success (Swamy, 1974). Takhtajan's (1969) theory of the origin of the angiosperms provides a reason for this failure and a solution to the problem. Studies on wood anatomy, leaves, sporophylls, and embryological structures all suggest a "neotenous" (or "progenetic" in the system of Gould, 1977) beginning for the flowering plants. In this view, selective pressures for early and rapid reproduction in pro-angiosperms led to early maturation and a consequent juvenilization of characters. Mature angiosperm structures, including the gametophytes, are typically homologous with immature gymnosperm counterparts. Even the angiosperm egg cell may not be a strict homologue with the ancestral gymnosperm egg, but rather with an early gametophytic cell that happened to be fertilizable in the evolutionarily novel circumstance of a precocious encounter with a sperm nucleus.

"Neoteny" may enhance the likelihood of double fertilization. If one immature gametophytic cell is fertilizable, it is not implausible that more than one would be. If the kin conflict theory is correct, "neoteny" also creates conditions necessary for selection to favor replacement of the gametophyte by the endosperm. Fertilization now occurs before the 
gametophyte is well-developed and prior to nutrient storage in the ovule. This allows an endosperm to start on roughly equal footing with the gametophyte and make a substantial contribution to the embryo. In addition, the delay between pollination and fertilization, months in most gymnosperms (Singh, 1978), is greatly reduced. This may decrease competition from later-arriving pollen, thereby increasing the probability that the embryo and endosperm have the same father.

Two questions are left unanswered by the kin conflict theory. First, the original double fertilization could have produced either a diploid or a triploid endosperm. Both should be selected to nourish the embryo more than the gametophyte does. The additional maternal chromosome complement could be a maternal strategy to reduce the selfishness of the endosperm by increasing its relatedness to other embryos (Willson \& Burley, in press) but I know of no way to test this. Second, the first endosperm might have been either embryonic or gametophytic in character since either a second embryo or a fertilized "gametophyte" could be pre-adapted for drawing additional nutrients into the seed.

Whatever the answers to these questions, the kin conflict theory does predict that the transition from gametophyte to endosperm was relatively gradual, with both functioning in the same ovules for a number of generations. Consider the contrary case in which the second fertilization essentially causes the gametophyte to become endosperm, so that only one functions as a nurse tissue in any given ovule. One would then expect maternal plants or gametophytes to evolve to prevent the second fertilization, thereby preventing development of the overly aggressive endosperm.

A gradual takeover by the endosperm minimizes this possibility. This is because the gametophyte would probably be selected to respond in an easier, but ultimately unsuccessful, way. As the endosperm begins to acquire excess (from the gametophytic viewpoint) nutrients, the gametophyte in the same ovule can respond by acquiring less, thus restoring its optimum. This strategy is probably realized more easily because selection could operate on pre-existing variation in gametophytic behavior, while the alternative of preventing the second fertilization without also preventing the first, would likely require entirely new mechanisms. But whenever the gametophyte restores its optimum, endosperms will be selected to acquire even more nutrients. Gametophytes can continue to respond by taking less until they are acquiring no resources at all. At this point, prevention of the second fertilization is no longer a selectable counterstrategy because it would leave the seed with no nutrient-procuring tissue.

Such a gradual replacement also seems likely on empirical grounds. In modern gymnosperms, and presumably the ancestor of the angiosperms, 
the early cleavage nuclei of the gametophyte all seem to continue to divide as the tissue develops (Singh, 1978). There is no obvious reason why precocious fertilization of one or more of these nuclei would prevent the others from dividing further. The result would be an endosperm and gametophyte both functioning in the same seed, setting the stage for the transition described above.

To summarize, the kin conflict theory requires three events in the early evolution of the endosperm: precocious fertilization, fertilization of egg and polar nuclei by identical sperm nuclei, and a transitory stage in which both endosperm and gametophyte function in the same ovules. All are in accord with our knowledge of the early evolution of the angiosperms.

\section{Alternative Hypotheses}

Several other hypotheses on the origin of the endosperm have been reported. One sees the endosperm as a product of male-female conflict, double fertilization being the male's way of becoming part of the apparatus for gaining maternal resources for his offspring (Charnov, 1979; Willson \& Burley, in press). The genetic basis for this conflict is really the same as in the kin conflict hypothesis, since in each the aggressiveness of the endosperm compared to the gametophyte is due to multiple paternity (Table 2). The hypotheses differ principally in the information assumed to be available to endosperm genes. Unlike the kin conflict model, the malefemale conflict hypothesis assumes that endosperm genes can be selected to act differently on the basis of their parentage, with alleles of paternal origin causing more aggressive action than the same alleles cause when maternally derived. This seems unlikely since it requires that identical alleles act differently and appropriately in the same cytological environment. When the frequency of an allele is low, the presence of another identical allele in the endosperm could serve as a cue of maternal origin (B. Charlesworth, personal communication) but this would become unreliable as the frequency increases.

In practice, these two hypotheses are difficult to separate since the genetic basis for conflict is the same. Tentative evidence against the male-female conflict hypothesis can be drawn from the results of reciprocal matings between species with different chromosome numbers. When the father is of the species with the higher chromosome number, the resulting endosperm will have a larger dose of paternal genes. The male-female conflict hypothesis therefore predicts that this cross will yield more aggressive endosperms and larger seeds than the reciprocal cross. In fact, the reciprocal cross usually produces larger and more viable seeds (Brink \& Cooper. 
1947). For this reason, and because of the simpler assumptions involved, I have concentrated on the kin conflict hypothesis, but most of the predictions in this paper are also predicted by the male-female conflict model.

Hypotheses not involving conflict of interest are more common. One view, that higher ploidy in the endosperm makes it a more efficient nutritive tissue than the gametophyte, is vulnerable because it cannot account for the participation of the male nucleus when higher ploidy could be achieved with maternal nuclei alone (Willson \& Burley, in press). The male nucleus could serve to make the nutritive tissue genetically and physiologically more compatible with the embryo (Nemec, 1910) but this could only come at the expense of compatibility with the maternal tissue from which the nutrients must come. The most complete non-conflict theory holds that double fertilization enhances the efficiency of the nutritive tissue through heterosis and by masking the effects of deleterious recessives (Brink \& Cooper, 1947).

Such factors may be among the proximal mechanisms that aid the endosperm in achieving its selective interests. But, as ultimate explanations, they seem inadequate. Too many questions remain unanswered unless kin-selected conflicts of interest are included. For example, in non-conflict theories, the severe reduction of the angiosperm gametophyte is often taken as a given, with the endosperm serving to compensate for its loss (Brink \& Cooper, 1947). But, why would the gametophyte shrink before there was something to replace it? Perhaps these theories could be modified for a gradual replacement of the gametophyte by a more efficient endosperm. Even so, if the endosperm evolved to alleviate some shortcoming of the gametophyte, why does it evolve past this point and completely replace the gametophyte? Only the kin conflict theory provides a simple explanation for why the endosperm and gametophyte cannot function in the same ovule.

Second, why is there no endosperm in the gymnosperms if double fertilization is an easy thing to evolve? The solution suggested above, that fertilization occurs too late for the endosperm to make a substantial contribution to the embryo, does not apply for all theories. If, for example, the biparental nature of the endosperm serves to bridge some physiological incompatibility of parent and embryo, it could do so even if the gametophyte acquired all the nutrients prior to fertilization. Brink \& Cooper (1947) argued that only in angiosperms does the embryo grow at the same time as the seed integuments, and therefore it needs the added vigor conferred by heterosis in the nutritive tissue. This argument assumes that the gametophyte is inadequate. However, while the embryo of gymnosperms does not grow at the same time as the integuments, the gametophyte does. 
If there were no conflict of interest, selection on maternal plants would produce integuments that take fewer nutrients and allow the gametophyte to get its share. An association of maternal plant and gametophyte should not be inadequate unless the assessment of what is adequate varies for the different seed components.

The third shortcoming of the non-conflict theories is their lack of explanation for the seemingly different roles played by the four seed components. Without conflict, any of the four could serve as the storage tissue and any of them could develop haustoria to absorb nutrients from other areas. But gametophytic and maternal tissues rarely do. Even if the haploidy of the gametophyte renders it incompetent, this cannot be true of the maternal tissues.

These objections also apply to non-adaptive explanations for the evolution of the endosperm. One cannot exclude the possibility that historical accident played a greater role than specifiable selective forces but this seems unlikely in the face of a well-supported adaptive hypothesis.

\section{Discussion}

On the basis of the available evidence, the kin conflict theory seems to provide the best account of both the origin of the endosperm and the current roles performed by the various parts of developing seeds. If this view is correct, it will affect research in several areas of biology.

The seed will have to be viewed not as a totally harmonious unit, but as an interdependent but sometimes contentious assemblage of relatives. Knowing how the selective goals of the seed components differ should be an aid to morphologists and physiologists in unravelling the process of seed development.

The theory also contributes towards a solution of Darwin's "abominable mystery", the origin of the flowering plants. Theories of a "neotenous" origin and the kin conflict theory are mutually reinforcing. The kin conflict theory requires a neotenous origin, and in turn provides an explanation for the evolution of the endosperm in terms of it. One important consequence is that one of the arguments for the angiosperms being monophyletic loses some of its force. In the absence of a convincing adaptive explanation, it has been considered unlikely that the endosperm could have evolved more than once (Stebbins, 1974). The kin conflict theory, while not actually supporting a polyphyletic interpretation, does at least raise the possibility that endosperms in different groups could be analogues which all arose in response to selective pressures for early and rapid reproduction. 
If the kin conflict theory is correct, it also means that parents cannot always enforce their preferred distribution of resources to offspring, a conclusion important to theories of parent-offspring conflict in animals as well as plants.

Finally, the evidence suggests that the theory of kin selection, crucial for the understanding of animal social behavior, is also important in what might be called the social behavior of plants. Kin selection should apply to any organism where conditions are such that it can reliably identify and affect the reproduction of relatives, as seems likely for the "individuals" involved in seed maturation. Indeed, as Charnov (1979) suggested, the endosperm may best be viewed as an individual that reproduces only through non-descendant relatives. Functionally, it is therefore closely allied to the sterile workers of many social insects.

\section{Note}

After submission of this manuscript, I received a copy of a manuscript from Mark Westoby and Barbara Rice (Evolution 36, 713) with some parallel ideas. They have independently derived the relatedness ratios in column 4 of Table 2 . See their paper for a somewhat different interpretation of how relatedness affected the evolution of the endosperm and for an excellent discussion of the evolution of the integuments as a barrier to embryos.

For helpful comments and criticism, I thank R. D. Alexander, J. L. Bronstein, N. Burley, B. Charlesworth, W. D. Hamilton, T. D. Price, B. J. Rathcke, W. H. Wagner, and M. F. Willson. M. F. Willson and N. Burley generously provided an unpublished manuscript. Some of the work was supported by a National Science Foundation graduate fellowship.

\section{REFERENCES}

Alexander, R. D. (1974). Ann. Rev. Ecol. Syst. 5, 325.

AleXANDER, R. D. \& ShERMAN, P. W. (1974). Science 196, 494.

BAKER, H. G. (1972). Ecology 53, 997.

BERLYN, G. P. (1972). In: Seed Biology (Kozlowski, T. T. ed.) Vol. 1, p. 223. New York: Academic Press.

Bhatnagar, S. P. \& Johri, B. M. (1972). In: Seed Biology (Kozlowski, T. T. ed.) Vol. 1, p. 77. New York: Academic Press.

Bhatnagar, S. P. \& Kallarackal, J. (1980). Cytologia 45, 247.

BrinK, R. A. \& COOPER, D. C. (1947). Bot. Rev. 8, 423; 9, 479.

Charnov, E. L. (1979). Proc. natn. Acad. Sci. U.S.A. 76, 2480.

ChOpRA, R. N. \& SACHAR, R. C. (1963). In: Recent Advances in the Embryology of Angiosperms (Maheshwari, P. ed.), p. 135. Delhi: University of Delhi. 
COE, G. E. (1954). Bot. Gaz. 115, 342.

COOK, R. E. (1981). Nat. Hist. $90,30$.

CUOPER, D. C. \& BRINK, R. A. (1949), Bot. Gaz. 111, 139.

DA VIS, G. L. (1966). Systematic Embryology of the Angiosperms. New York: Wiley.

DNyANSAGar, V. R. (1954). J. Indian bot. Soc. 33, 433.

FAGERLIND, F. (1936). Bot. Notiser 1936, 577.

Favre-duchartre, M. (1958). Phytomorphology 8, 377

GHOSH, M. (1954), Curr. Sci. 23, 24.

Gould, S. J. (1977). Ontogeny and Phylogeny. Cambridge: Belknap Press.

HAMILTUN, W. D. (1964). J. theor. Biol. 7, 1; 7, 17.

Hamilton, W. D. (1972). Ann. Rev. Ecol. Syst. 3, 193.

HJELMQVist, H. (1954). Phytomorphology 14, 186.

JoHRI, B. M. (1963). In: Recent Advances in the Embryology of Angiosperms (Maheshwari, P. ed.), p. 69. Delhi: University of Delhi.

Johri, B. M. \& Bhatnagar, S. P. (1969). Rev. Cytol. Biol. veg. 32, 353.

JOHRI, B. M. \& PRAKASH, S. (1965). Phytomorphology 4, 173.

KRESS, W. J. (1981). Syst. Bot. 6, 101.

KuIJT, J. (1969). Biology of Parasitic Flowering Plants. Berkeley: University of California Press.

Linskens, H. F. (1969). In: Fertilization: Comparative Morphology, Biochemistry and Immunology (Metz, C. B. \& Monroy, A. eds), Vol. 2. New York: Academic Press.

Macnair, M. R. \& PARKer, G. A. (1978). Anim. Behav. 26, 111.

MaCnair, M. R. \& Parker, G. A. (1979). Anim. Behav. 27, 1202.

MALECOT, G. (1948). Les Mathematiques de l'Heredite. Paris: Masson et Cie.

MASAND, P. \& KAPIL, R. N. (1966). Phytomorphology 16, 158.

MeEuse, A. P. (1963). Phytomorphology 13, 237.

Michod, R. E. \& HAMILTON, W. D. (1980). Nature. Lond. 288, 694.

Mukrada, A. J. (1969). New Phytol. 68, 1145.

NAGL, W. (1978). Endopolyploidy and Polyteny in Differentiation and Evolution. Amsterdam: North Holland.

Nakayana, R. (1954). Phytomorphology 4, 173.

Narayana, R, (1958). Phytomorphology 8, 146.

NEMEC, B. (1910). Das Problem der Befruchtungsvorgange und andere zytologische Fragen. Berlin. cited in: P. Maheshwari (1950). Introduction to the Finbryology of the Angiosperms. New York: McGraw-Hill.

NyGRen, A. (1967). In: Encyclopedia of Plant Physiology XVIII (Ruhland, W. ed.), p. 551. Berlin: Springer-Verlag.

OSIER, G., ESCHEL, I. \& COHEN, D. (1977). Theor. pop. Biol. 12, 49.

Parker, G. A. \& MaCnair, M. R. (1978). Anim. Behav. 26, 97.

Parker, G. A. \& Macnalr, M. R. (1979). Anim. Behav. 27, 1210.

Prakash, S. (1960). Phytomorphology 10, 224.

SiNGH, H. (1978). Embryology of Gymnosperms. Berlin: Gebruder Borntraeger.

Singh, H. \& Johri, B. M. (1972). In: Seed Biology (Kozlowski, T. T. ed.), Vol. 1, p. 21. New York: Academic Press.

Stramps, J. A., Metcalf, R. A. \& KRIShnan, V. V. (1978). Behav. Ecol. Sociobiol. 3, 369

Stebrins, G. L. (1974). Flowering Plants: Etolution Above the Species Level. Cambridge: Belknap Press.

Stephenson, A. G. (1981). Ann. Rev. Fcol. Syst. 12, 253.

SWAMY, B. G. L. (1949a). Am. Midl. Nat. 41, 184.

SWamy, B. G. L. (1949b). Am. Midl. Nat. 41, 203.

SINAMY, B. G. L. (1974). Phytomorphology 14, 186.

Takhtajan, A. (1974). Flowering Plants: Origin and Dispersal. London: Oliver \& Boyd. Trivers, R. L. (1974). Am. Zool. 14, 249.

TRIVERS, R. L. \& HARE, H. (1976). Science 191, 249. 
West Eberhard, M. J. (1975). Quam. Rev. Biol. 50, 1.

WESTOBY, M. (1981). Am. Nat. 118, 882.

Willson, M. F. \& Burley, N. (in press). Mate Choice in Plants: Mechanisms and Con sequences. Princeton: Princeton University Press.

WILsON, E. O. (1975). Sociobiology: The New Synthesis. Cambridge: Belknap Press. 